\title{
VE-cadherin promotes vasculogenic mimicry by modulating kaiso- dependent gene expression
}

\author{
Daniel Delgado-Bellido ${ }^{1,2}$ - Mónica Fernández-Cortés ${ }^{1,2}$ - María Isabel Rodríguez ${ }^{3}$ Santiago Serrano-Sáenz ${ }^{1,2}$. \\ Arkaitz Carracedo $^{2,4} \cdot$ Angel Garcia-Diaz $^{1} \cdot$ F Javier Oliver $^{1,2}$
}

Received: 13 March 2018 / Revised: 25 April 2018 / Accepted: 27 April 2018 / Published online: 21 May 2018

(c) ADMC Associazione Differenziamento e Morte Cellulare 2018

\begin{abstract}
Aberrant extra-vascular expression of VE-cadherin (VEC) has been observed in metastasis associated with vasculogenic mimicry (VM); however, the ultimate reason why non-endothelial VEC favors the acquisition of this phenotype is not established. In this study, we show that human malignant melanoma cells have a constitutively high expression of phoshoVEC (pVEC) at Y658; pVEC is a target of focal adhesion kinase (FAK) and forms a complex with p120-catenin and the transcriptional repressor kaiso in the nucleus. FAK inhibition enabled kaiso to suppress the expression of its target genes and enhanced kaiso recruitment to KBS-containing promoters. Finally we have found that ablation of kaiso-repressed genes WNT11 and CCDN1 abolished VM. Thus, identification of pVEC as a component of the kaiso transcriptional complex establishes a molecular paradigm that links FAK-dependent phosphorylation of VEC as a major mechanism by which ectopical VEC expression exerts its function in VM.
\end{abstract}

\section{Introduction}

Solid tumors require blood vessels for growth, and access to oxygen and nutrients, and anti-angiogenic therapies are designed to target vascular endothelial cells (ECs) to form tumor blood vessels. Although numerous preclinical models have recognized the efficient use of angiogenesis inhibitors to limit tumor growth, collectively only a growth delay has

\section{Edited by H. Ichijo.}

Electronic supplementary material The online version of this article (https://doi.org/10.1038/s41418-018-0125-4) contains supplementary material, which is available to authorized users.

\section{Angel Garcia-Diaz}

agdiaz@ipb.csic.es

$\triangle$ F Javier Oliver

joliver@ipb.csic.es

1 Instituto de Parasitología y Biomedicina López Neyra, CSIC, Granada, Spain

2 CIBERONC, Instituto de Salud Carlos III, Madrid, Spain

3 Centro Pfizer-Universidad de Granada-Junta de Andalucía de Genómica e Investigación, Oncológica (GENYO), Granada, Spain

4 CIC bioGUNE, Derio, Spain been achieved in the clinic [1]. In 1999, Maniotis et al. [2] presented a new interpretation of previous findings describing cancer cells covering non-endothelial vascular channels that contained red blood cells. This was the initial report defining tumor cell vasculogenic mimicry (VM) as the de novo formation of perfusable, matrix rich, vasculogenic-like network in three-dimensional (3D) matrix in vitro, which resembled the matrix-rich network observed in aggressive tumors in patients [3]. The plasticity/ embryonic-like phenotype of certain types of cancer cells could explain their ability to mimic the activities of ECs and to participate in processes such as neovascularization and the formation of a fluid-conducting, matrix-rich meshwork $[4,5]$. The presence of VM is associated with a high tumor grade, short survival, invasion, and metastasis. ECs express various members of the cadherin superfamily, in particular vascular endothelial (VE-) cadherin (VEC), which is the main adhesion receptor of endothelial adherent junctions. Aberrant extra-vascular expression of VEC has been observed in certain cancer types associated with VM [6].

VEC is a trans-membrane protein commonly expressed in the endothelium, where it is responsible for cell-cell adhesion [7]. Although VEC used to be considered specific for ECs, its expression has been strongly associated with aggressiveness and VM in melanoma. Interestingly, VEC 
can be found in highly aggressive tumor cells but not in non-aggressive ones. Moreover, its downregulation in melanoma implied the loss of VM formation [8]. EC barrier function is mediated in part by homotypic binding of transmembrane adherent junction proteins such as VEC [7]. Post-translational VEC modifications trigger junctional changes, VEC internalization, and increased vascular permeability, which can modulate tumor cell intravasation and extravasation [9, 10]. Focal adhesion kinase (FAK) is a cytoplasmic tyrosine kinase co-activated by integrin and VEGFR-2 receptors in the control of vascular permeability [11]. Small-molecule FAK inhibitors prevent tumor progression in mice [12] and are being evaluated in clinical trials [13]. FAK expression and activation are also elevated in ECs associated with malignant astrocytoma, ovarian tumors, and aggressive melanoma [14-16]. Recent studies have shown that the VECY658 residue is a target of FAK in tumor-associated ECs, and inhibition of endothelial FAK activity prevents tumor metastasis by enhancing barrier function [17].

In the current study, we have elucidated the mechanistic link leading from elevated pVEC expression to induction of $\mathrm{VM}$ in metastatic melanoma through the analysis of intracellular dynamic and functional consequences of the elevated expression of pVEC (Y658). These results establish a molecular paradigm associating pVEC (Y658) with VM transformation of melanoma cells owing to its ability to modulate p120 nuclear localization and kaiso-dependent gene expression.

\section{Material and methods}

\section{Reagents and antibodies}

The following reagents were used: PF-562271 (PF-271, Abcam), VEGF (Peprotech), sodium pervanadate V2O5 + $6 \mathrm{NaOH} \rightarrow 2 \mathrm{Na} 3 \mathrm{VO} 4+3 \mathrm{H} 2 \mathrm{O}$ (5), Corning Matrigel Basement Membrane Matrix for in vitro angiogenesis experiments. Antibodies used were: Y658 VEC rabbit (1:1000 WB, 1:100 IF, Thermofisher), VEC C-ter mouse (1:500 WB, 1:50 IF, $2 \mu \mathrm{g}$ IP, clone F-8, sc-9989), FAK rabbit (1:2000 WB, clone C-20, sc-558), VEC extracellular domain mouse (1:1000 WB, 1:100 IF, clone BV6, Millipore), anti-phosphotyrosine p-Tyr mouse (1:500 WB, $2 \mu \mathrm{g}$ IP, clone PY20, sc-508), kaiso mouse (1:1000 WB, clone $6 \mathrm{~F}$, Millipore), kaiso ChIP grade mouse ( $8 \mu \mathrm{g}$ ChIP, clone $6 \mathrm{~F} 8$, Abcam), $\alpha$-tubulin mouse $(1: 10000 \mathrm{WB}$, clone B-5-12, Sigma-Aldrich), p120 catenin mouse (1:1000 WB, 1:100 IF, BD Biosciences), lamin B1 rabbit (1:1000 WB, Abcam), PARP-1 mouse (1:1000 WB, Calbiochem), Y731 VEC rabbit (1:1000 WB, Assay Biotech A8251), and Glut1 rabbit (1:1000 WB Santacruz H-43).

\section{Cell lines and construction of GFP-tagged VEC}

Human melanoma cells MUM 2B, MUM 2C, C8161, and C81-61 were grown in RPMI medium supplemented with $10 \%$ fetal bovine serum, $2 \mathrm{mM}$ of L-glutamine, and $1 \%$ penicillin/streptomycin (PAA laboratories). Human umbilical vein endothelial cells (HUVEC) were grown in endothelial cells growth medium-2 (EGM-2) (Lonza). All cells were cultured at $37{ }^{\circ} \mathrm{C}$ and $5 \% \mathrm{CO}_{2}$ in incubator cells. cDNA of human VEC fused in-frame with GFP at the COOH-terminus (VEC-GFP), VEC Y658F and Y658E was a kind of gift from Dr. Masahiro Murakami. These constructs were subcloned into pcDNA3.1 (Invitrogen).

\section{In vitro angiogenesis assay}

The effect of FAK-Inhibitors, siVE-cadherin (siVEC), VECad K.O, WT, and Y658F on the formation of tube-like structures in Matrigel (BD Biosciences) was determined according to manufacturer's instructions. Briefly, 96-well plates were coated with $50 \mu \mathrm{l}$ of BD Matrige ${ }^{\mathrm{TM}}$ Basement Membrane Matrix and allowed to solidify at $37^{\circ} \mathrm{C}$ in $5 \%$ $\mathrm{CO}_{2}$ for $30 \mathrm{~min}$. Cells were treated with PF-271 $(1 \mu \mathrm{M})$ for $24 \mathrm{~h}$ or Scb, siFAK, siCCND1, siWNT11, siVEC transfected for $48 \mathrm{~h}$ as described previously. After 24 or $48 \mathrm{~h}$, respectively, of incubation, images were acquired using an Olympus CKX41 microscope. The formation of tube-like structures was then quantified by Wimasis program. Each treatment was performed in triplicate, and the experiments were independently repeated at least three times.

\section{Flow cytometry}

Cells were plated in 6-well plates at $1.5 \times 10^{5}$ seed density per well. After $24 \mathrm{~h}$ of PF-271 treatment, the cells were washed with cold phosphate buffered saline (PBS), fixed with $70 \%$ cold ethanol, and stained with propidium iodide $10 \mu \mathrm{g} / \mathrm{mL}$ supplemented with ribonuclease A $100 \mu \mathrm{g} / \mathrm{mL}$ (Sigma). Cell cycles were determined by FACSCalibur flow cytometer and analyzed with FlowJO 7.6.3 software (Tree Star).

\section{Quantitative RT-PCR}

Total RNA was isolated by RNeasy Mini Kit (Qiagen) according to the manufacturer's recommendations. About 1 $\mu \mathrm{g}$ of RNA from each sample was treated with DNase I, RNasinRibonuclease inhibitors (Invitrogen) and reversetranscribed using iScriptcDNA synthesis kit (Biorad) following the manufacturer's protocols. cDNA was amplified using the iTaq Universal SYBR green supermix (Biorad). Each reaction was performed in triplicate using CFX96 
A

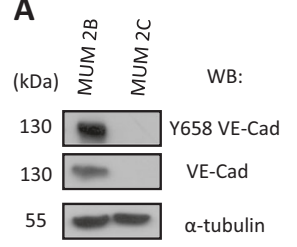

C

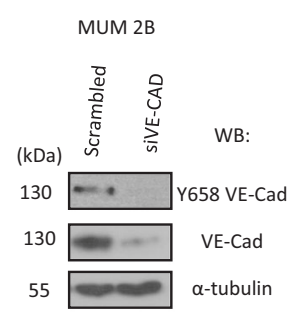

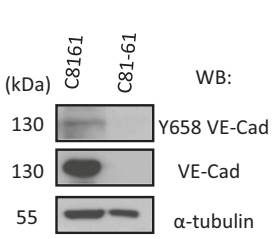

D

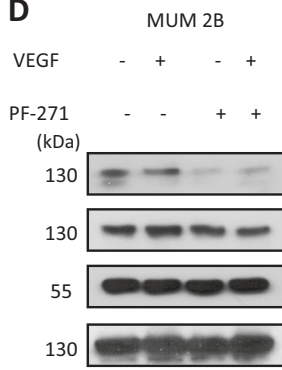

B
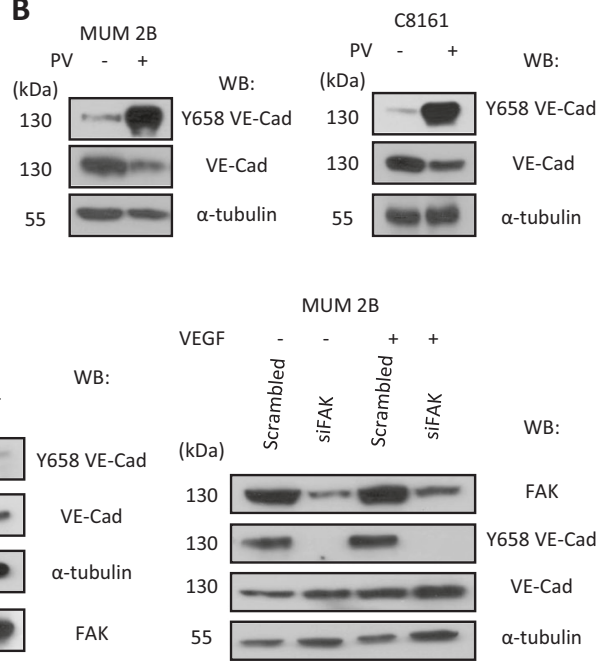

Fig. 1 Elevated expression of phospho-VE-cadherin in aggressive melanoma cells: a VE-cadherin and Y658 VE-cadherin expression in aggressive melanoma cells (MUM 2B, C8161) and poorly melanoma cells (MUM 2C, C81-61), (b) pervanadate treatment in MUM 2B and C8161 showed an increase in Y658 expression levels with decrease in

Real-time PCR detection systems. Primer sequences for the targets and the annealing temperature $\left(60^{\circ} \mathrm{C}\right)$ are the following: 36B4: Forward 5'-CAGATTGGCTACCCAAC TGTT-3', Reverse 5'-GGCCAGGACTCGTTTGTACC-3'; CCND1: Forward 5'-CCGTCCATGCGGAAGATC-3', Reverse 5'-GAAGACCTCCTCCTCGCACT-3'; WNT11: Forward 5'-GCTTGTGCTTTGCCTTCAC-3', Reverse 5'-TGGCCCTGAAAGGTCAAGTCTGTA-3'. Statistical analyses were conducted using Graph Pad Prism software. Statistical significance was calculated using a Student's $t$ test (unpaired, two-tailed) with measurements from at least three independent trials.

\section{Chromatin immunoprecipitation (ChIP)}

Cells were grown to approximately $80-90 \%\left(18 \times 10^{6}\right.$ cells per IP) confluence with PF-271 $1 \mu \mathrm{M}$ for $24 \mathrm{~h}$, siVEC for 48 $\mathrm{h}$ with the respective controls. Culture medium was aspirated and cells were washed twice with cold-PBS. ChIP was performed following SimpleChIP Enzymatic Chromatin IP Kit (Magnetics beads) (Cell signaling). qPCR promoter specific primers used: CCND 1 promoter (15): Forward 5'TTTACATCTGCTTAAGTTTGCG-3', Reverse 5'-TTA GAATTTGCCCTGGGACT-3'; WNT 11 promoter (31): Forward 5'-CACCCTTCCCACTTCCAA-3', Reverse 5'GAGAGACGTCTGCTTGGCT-3'; PSMD5 gene promoter as a positive control based on public available ZBTB33 ChIP-seq data (GSM1334009 and GSM803504) (15): Forward 5'-GATCTGATCACAGCCTCCTTG-3', Reverse: 5'-CAACCACTGGCTACAGTTGA-3' and negative control RPL30 with irrelevant sequence.
VE-cadherin expression, (c) silencing VE-cadherin proves that Y658 VE-cadherin antibody is specific in this melanoma models, (d) VEGF $(80 \mathrm{ng} / \mathrm{ml})$ for $30 \mathrm{~min}$ treatment with or without PF-271 $1 \mu \mathrm{M}$ or siFAK for $24 \mathrm{~h}$ or $48 \mathrm{~h}$ showed a total decrease in the expression of Y658 VE-cadherin

\section{Gene editing}

MUM2B knockout (ko) cells for the VE-Cad gene were generated using the CRISPR-Cas9 technology. Five different sgRNAs were designed using the Zhang Lab Optimized CRISPR design tool [18] and cloned into the pL-CRISPR.EFS.GFP which was purchased from the Addgene public repository (\#57818). sgRNA guides were validated in HEK293T Cells using the GeneArt Genomic Cleavage Detection Kit (Invitrogen, Carlsbad, USA) according to the manufacturer's instructions. Lentiviral particles for the best two sgRNAs in terms of allelic disruption (GGCAGGCGCCCGATGTGGCG and GATGATGCTCCTCGCCACATC) were produced as previously described [19].

MUM2B cells were co-transduced at MOI 10-100 with the two different lentiviral particles, and an efficiency of transduction close to $100 \%$ was confirmed by Flow Cytometry. Finally, allelic disruption and protein truncation were confirmed by Sanger sequencing and western blot (Fig. 8a). Cellular pools of the $k o$ cells were used for the in vitro angiogenesis formation assays (Fig. 8c).

\section{Results}

\section{Elevated expression of pVEC (Y658) in aggressive melanoma cells}

Aberrant extra-vascular expression of VEC has been observed in certain cancer types associated with VM. We 
MUM 2B

A

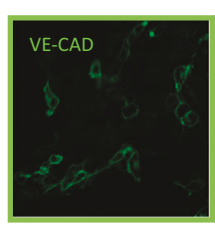

Control
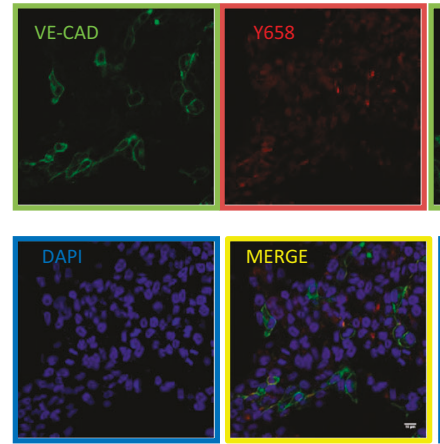

PF-271
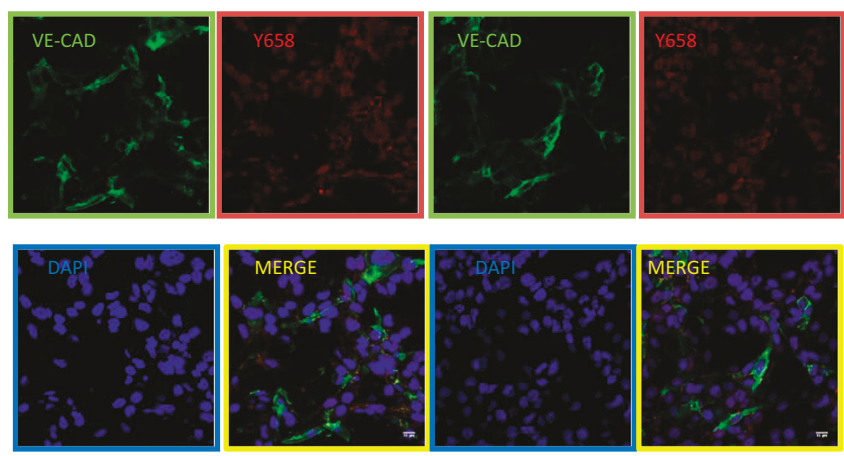

Fig. 2 Pharmacological focal adhesion kinase (FAK) inhibition and genetic FAK inhibition through small interfering siRNA decreased directly the expression of pY658 VE-Cad in aggressive melanoma cells VE-Cad positive and nuclear Y658 VE-cadherin translocation: a Immunofluoresence of Y658 VE-cadherin (red), VE-cadherin (green),

have previously shown that most of the VEC present in melanoma cells that develop VM is in the phosphorylated form [20]. In the current study, we focused on pVEC (Y658) because this tyrosine is highly conserved among species and has been reported to be phosphorylated by permeability-increasing agents such as VEGF [17]. Total VEC expression was measured in different melanoma cell lines from either cutaneous or uveal origin. As shown in Fig. 1, while the non-aggressive uveal melanoma cell line MUM 2C did not express VEC, the highly aggressive cell lines MUM 2B (uveal) and C8161 (cutaneous) expressed high levels of VEC (Fig. 1a). This VEC was indeed highly phosphorylated as revealed after inhibition of phosphatases and western blot with anti-pVEC (Y658) (Fig. 1b). Note that pVEC (Y731) was not detected in MUM2B (compared with ECs HUVEC) in Fig. S1A). VEC expression was further corroborated by silencing VEC through siRNA (Fig. 1c). To confirm the specificity of the antibody against pVEC (Y658), we transfected different constructs expressing wtVEC, the phosphomimetic VEC mutant (Y658E) and the non-phosphorylable VEC mutant (Y658F) [21] in MUM2C (cells that do not express endogenous VEC (Fig. S1B). pVEC was only detected in cells transfected with the
MUM 2B
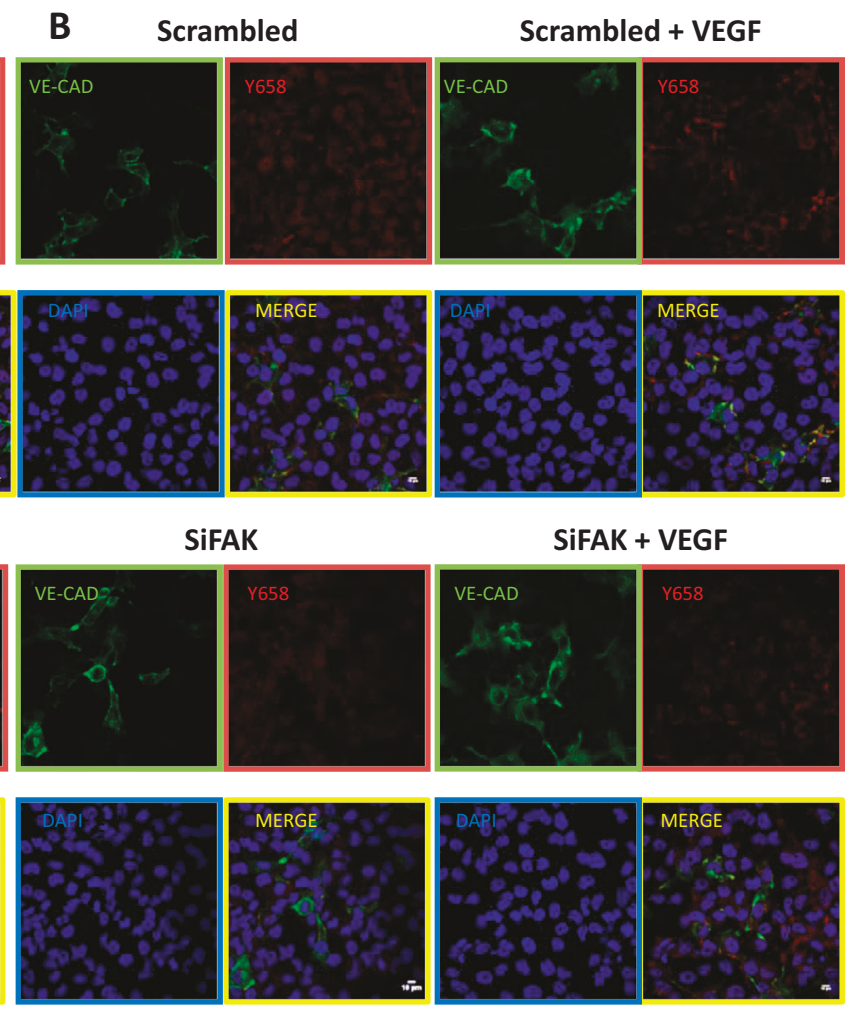

and DAPI (nuclear stain, blue) with VEGF $+/-$ PF-271 $1 \mu \mathrm{M}$ or siFAK, (b) in aggressive melanoma cells MUM 2B showed that inhibition of the activity of FAK produces Y658 VE-Cad decrease; bars $15 \mu \mathrm{m}$

Y658E mutant (Fig. S1B). Using the anti-pVEC (Y658) antibody, VEC signal did not co-localize (merged) with GFP-expressing cells when the non-phosphorylable (Y658F) protein was transfected (Fig. S1 C), further confirming the specificity of the antibody.

Recent studies have shown that the Y658 residue of VEC is a target of FAK in tumor-associated ECs, and identify FAK as a key regulator of EC barrier function controlling tumor metastasis [22]. To investigate the role of FAK signaling in VM, we used a specific FAK inhibitor (PF-271). Treatment with PF-271 in the presence or absence of VEGF-A resulted in decreased pVEC Y658 phosphorylation independently of VEGF-A (Fig. 1d, left). Moreover, siRNA of FAK completely prevented Y658 phosphorylation (Fig. 1d, right). To determine the effect of PF-271 on the level of phosphorylation, a co-immunoprecipitation was performed using anti pY antibody and western blot of FAK and VE-Cadherin (Fig. S2A). Indeed, specific inhibition of Y397 FAK phosphorylation (but not of Y416c-Src phosphorylation) has been recently demonstrated [17, 23] without affecting phosphorylation of Y576 FAK.

This finding was corroborated by indirect immunofluorescence of Y658 and VEC under the same 
Fig. 3 pY658 VE-cadherin participates in p120 catenin nuclear import and forms a complex with p120 catenin and the transcription repressor kaiso: a Subfractionation cytosol (CE)- nucleus (NE) scrambled/ siFAK showed a VE-cadherin nuclear and this internalization is suppressed with the inhibition of FAK as wells as Y658 VEcadherin and p120. b

Subfractionation cytosol (CE)- nucleus (NE) control/PF271 showed the same results. $\mathbf{c}$ Silencing VE-cadherin prevents the internalization of p120 to nucleus and chromatin. $\mathbf{d}$ Silencing VE-cadherin prevents the internalization of p120 to the nucleus. e Subfractionation cytosol (CE)- nucleus (NE) control/PF-271 with Leptomycin B $(20 \mathrm{ng} / \mathrm{ml})$
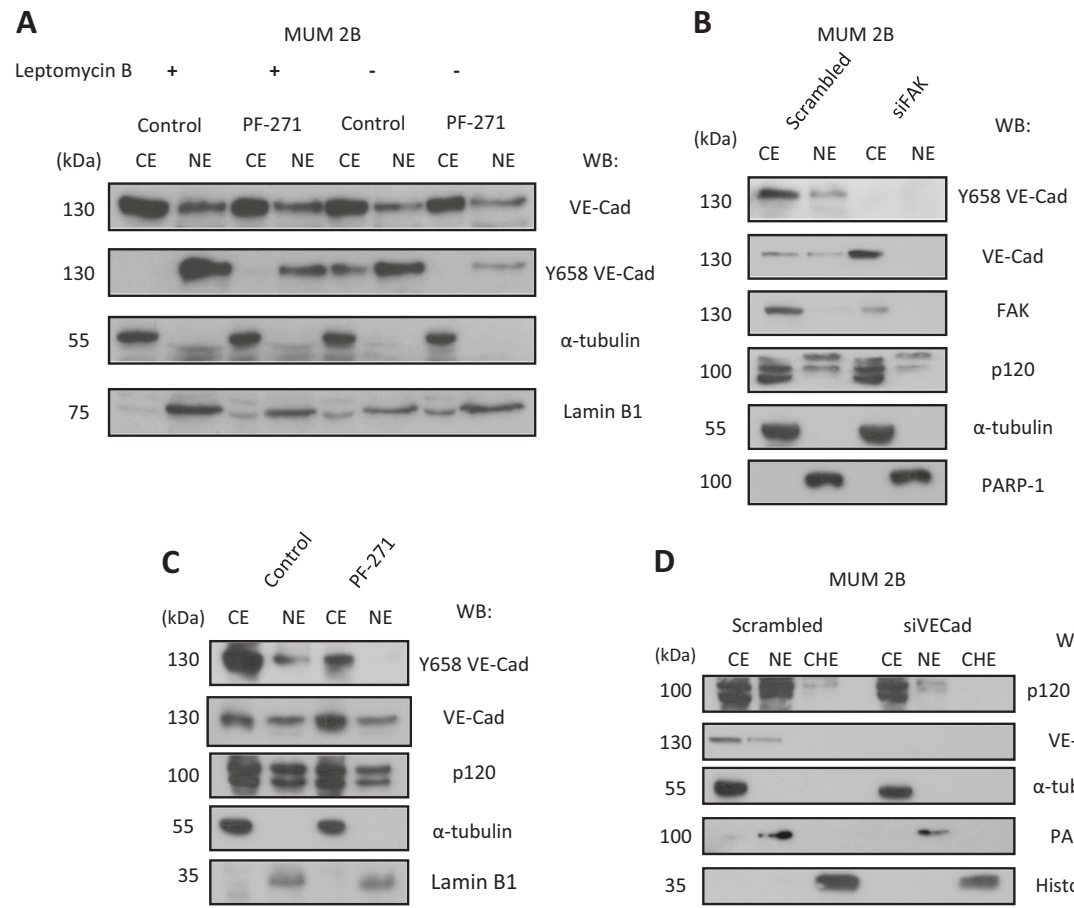

D

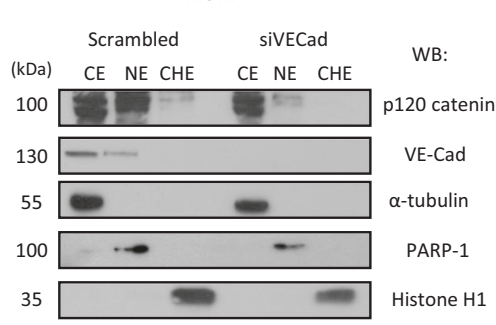

experimental conditions (Fig. 2a). VEGF-A treatment lead to an increase in pVECY658 that was prevented by either disabling FAK activity or FAK knockdown (Fig. 2a, b). These results suggested that elevated pVEC could be attributed to a permanent FAK activation, and in a lesser extent, to the engagement of VEGF to its receptor.

To further substantiate this aberrant VEC behavior in malignant melanoma cells, we used a different cell line, C8161 (cutaneous malignant melanoma cell line) which has also been shown to form VM channels; indeed, these cells displayed the same characteristic as MUM 2B in terms of VEC expression and elevated levels of pVECY658; similar results were also obtained after siFAK in a subcellular fractionation experiments (Fig. S3 A).

\section{pVEC (Y658) forms a complex with p120 catenin and participates in its nuclear import}

To confirm the presence of nuclear pVEC (Y658), we used Leptomycin B (to inhibit nuclear export) with PF-271 which led to accumulation of Y658 in the nucleus (Fig. 3a).

VEGF-A promotes vascular permeability by weakening adherent junctions and tight junctions, resulting in transient opening of the endothelial cell-cell contacts [24]. In fact, VEGF-A promotes tyrosine phosphorylation of VEC and its binding to different partners: $\beta$-catenin, plakoglobin, and p120, via Src-dependent mechanism [25]. Moreover, VEGF signaling reduces the association between VEC and p120catenin promoting clathrin-dependent VEC endocytosis;
[24] binding of p120 to VEC prevents its internalization, while p120 silencing leads to degradation of VEC, and loss of cell-cell contacts [26]. Indeed, while treatment of HUVEC with VEGF prevented VEC association with p120-catenin (Fig. S4A, left panel), this complex remained in MUM 2B irrespective of VEGF treatment (Fig. S4A, right panel).

To get additional information on how FAK impacts the stability of this complex in MUM 2B cells, we performed siFAK experiments and we found that siFAK completely prevented the nuclear localization of VEC and promoted its accumulation in the cytosol (Fig. 3b). Similar results were obtained after treatment with the FAK inhibitor PF271 (Fig. 3c). In both cases, nuclear p120 diminished after FAK disabling (fourth lane in Fig. 3a, b). Indirect immunofluorescence also revealed the co-localization (merged signal) of p120 and VEC in the nucleus which was blunted after FAK inhibition (Fig. S3E, see also S3E enlargements). It is well recognized that p120, like the classical betacatenin, undergoes nucleo-cytoplasmic trafficking and regulates gene expression [27]. To have a first evidence of the biological significance of the presence of pVEC (in complex with p120) in the nucleus, we silenced VEC and performed a subcellular fractionation assay (including the chromatin fraction) to localize p120. The results in Fig. 3d show that VEC (in phosphorylated form) was needed to fully recruit p120 to chromatin. Globally these results show that nuclear pVEC was needed to fine tune p120 recruitment to chromatin, opening the possibility to impact on p120regulated gene expression. 

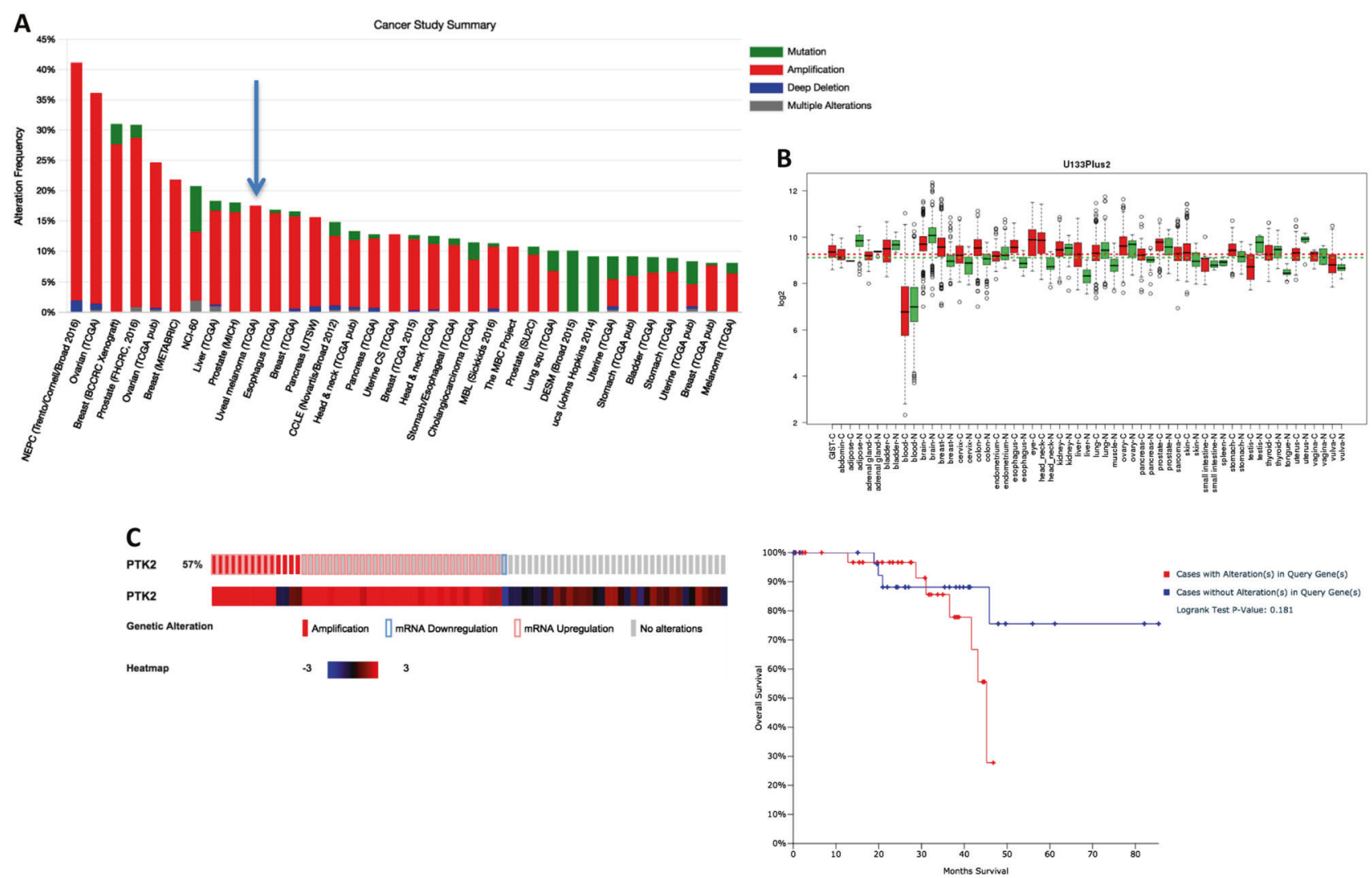

Fig. 4 Genetic alterations in FAK and FAK mRNA expression in uveal melanoma patients: a Bar graph showing the percentage of alteration of PTK2 (FAK) gene in different types of cancer (cBioportal). b Genetic alterations affecting uveal PTK2 (FAK) in uveal melanoma; $57 \%$ of cases presented either genetic or mRNA alterations. The correlation between PTK2 mRNA expression and patients' overall survival (c) in uveal melanoma patients

\section{Genetic alterations in FAK and FAK mRNA expression in uveal melanoma patients}

All the above results suggested that the ultimate reason to explain the elevated basal VEC phosphorylation in malignant uveal melanoma cells was due to constitutively active FAK. By analyzing the cBioPortal database, a platform of 48,333 tumor samples, we found that the copy number of the FAK gene was highly amplified in numerous human cancers, such as ovarian, breast, prostate, and uveal melanoma (Fig. 4a). Moreover, we used a web database of Gene Expression across Normal and Tumor tissues (GENT) that provides gene expression patterns through more than 34,000 samples. The FAK gene was particularly overexpressed (the highest of all studies in the database) in uveal melanoma (Fig. 4b). Furthermore, 57\% of Uveal Melanoma patients included in the study of TCGA (80 samples) displayed either gene alteration (amplifications) or mRNA upregulation (Fig. 4c), suggesting that FAK regulates normal cell growth in vivo. Moreover, we found that alterations in FAK expression corresponded with a clear tendency (although not statistically significant, probably due to the relatively low number of patients) to a decreased overall survival (Fig. 4c right).

\section{Abrogation of VM through pharmacological inhibition of FAK and siFAK}

In view of the elevated expression of FAK in uveal melanoma samples leading to high levels of constitutive pVEC (Y658) in malignant melanoma cells, we investigated the cell's capacity to form de novo vasculogenic-like network in 3D matrix (Matrigel) after modulation of the upstream kinase FAK; results in Fig. 5 show that FAK inhibitor (PF271) or siFAK abolished VM formation as measured by the capacity to form loops quantified by Wimasis (Fig. 5a-d); siFAK was confirmed by western blot (Fig. 5e).

\section{pVEC (Y658) regulates the transcriptional repressor kaiso and restrains the binding of kaiso to their target genes CCND1 and Wnt 11}

The transcription factor kaiso is a member of the $\mathrm{BTB} / \mathrm{POZ}$ subfamily of zinc finger proteins (POZ-ZF); kaiso 
A
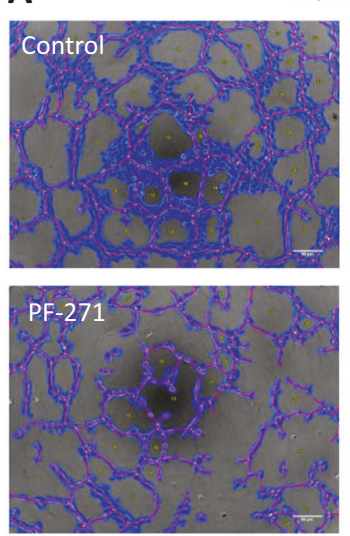

MUM 2B
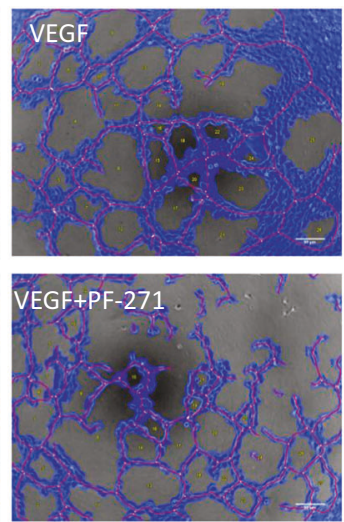

B
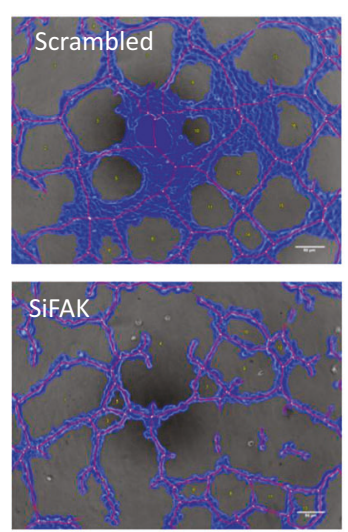

MUM 2B

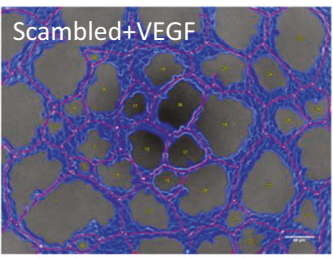

SIFAK+VEGF

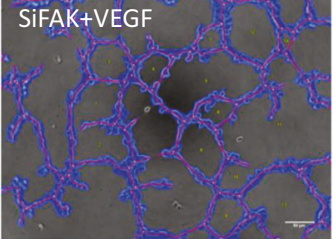

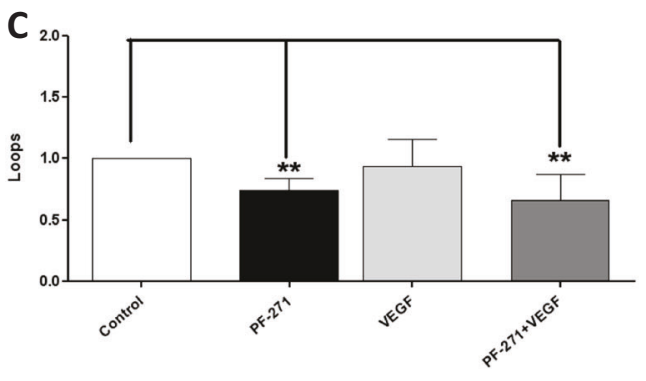

Fig. 5 Abrogation of vasculogenic mimicry through pharmacological inhibition of FAK and SiFAK: a, b In vitro angiogenesis assay with Matrigel in MUM 2B showed the effect of FAK-inhibitors (PF-271 and SiFAK) with or without VEGF, images were acquired using an Olympus CKX41 microscope (Bars $50 \mu \mathrm{m}$ ) and $\mathbf{c}, \mathbf{d}$ the formation of

recognizes and binds a specific DNA consensus sequence (kaiso binding sites KBS, TCCTGCnA, as a transcriptional repressor), as well as methylated $\mathrm{CpG-dinucleotides} \mathrm{[27].}$ Previous studies have shown that kaiso forms a complex with p120 and this complex prevent the binding of kaiso to its target genes promoters: CCDN1, WNT 11, MMP-7, and others [28-30]. Using a coIP approach, we show that VEC is also part of this complex with p120/kaiso (Fig. 6 a, b); moreover, abrogation of VEC phosphorylation, through FAK inhibition (PF-271), diminished complex formation and its nuclear import (Fig. 6 a-b). Interestingly, depletion of pVEC (Y658) forced the accumulation of VEC and p120 in the cytoplasmic fraction.

As a first approach to evaluate the impact of pVEC (Y658) in kaiso-dependent gene expression, we measured WNT11 and CCDN1 mRNA after either PF271 treatment or siFAK; both treatments, indeed, induced a significant reduction of WNT11 and CCDN1 mRNA levels (Fig. 7 a, b). Similarly, siVEC elicited a strong downregulation in the expression of both genes suggesting that kaiso was recruited more efficiently (then activating its repressor activity) after preventing Y658 phosphorylation or removing VEC expression.

We further analyzed the consequences of preventing VEC phosphorylation in the recruitment of kaiso to KBS by performing chromatin IP. Both PF271 treatment or siVEC robustly increased kaiso binding to the WNT11 and CCND1 promoters (Fig. 7c, d) then negatively regulating kaiso-repressor activity, as shown in Fig. 6a. To confirm the relationship between the increased expression of kaisodependent genes and VM, we performed in vitro VM formation after silencing CCDN1 or WNT11 which resulted in abrogation of vasculogenesis activation (Fig. 8a-d).

\section{VEC/Y658 phosphorylation is essential in the ability to form VM}

To further substantiate the impact of Y658 of VEC in the capacity to form VM, we performed an in vitro angiogenesis assay in Matrigel in MUM 2B cells permanently ko for VEC after gene editing with CISPR/Cas9, which were restored with either wtVEC or the mutant/non-phosphorylable VEC (Y658F) (Fig. 9). The depletion of VEC in MUM 2B was corroborated by western blot (Fig. 9a); siVEC silencing blunted VM capacity (Fig. 9b) in a similar way than CRISPR-depleted VEC cells (Fig. 9b, c). The rescue of VEC ko cells with wtVEC restored VM capacity (Fig. 9c, d), contrary to what was observed after the re-introduction of the mutant VEC (Y658F) in VEC ko cells (Fig. 9c, d). Overall these results suggest that Y658 phosphorylation of 
Fig. 6 pY658VE-cadherin represses the binding of kaiso to their target genes CCND1 and Wnt 11: a,b Co-

immunoprecipitation of VEcadherin (A) or p120 (B) (Control/PF-271) demonstrated the presence of a complex union of VE-cadherin/p120/kaiso and that this interaction is phosphorylation of $\mathrm{Y} 658$ dependents in MUM 2B and C8161 cells
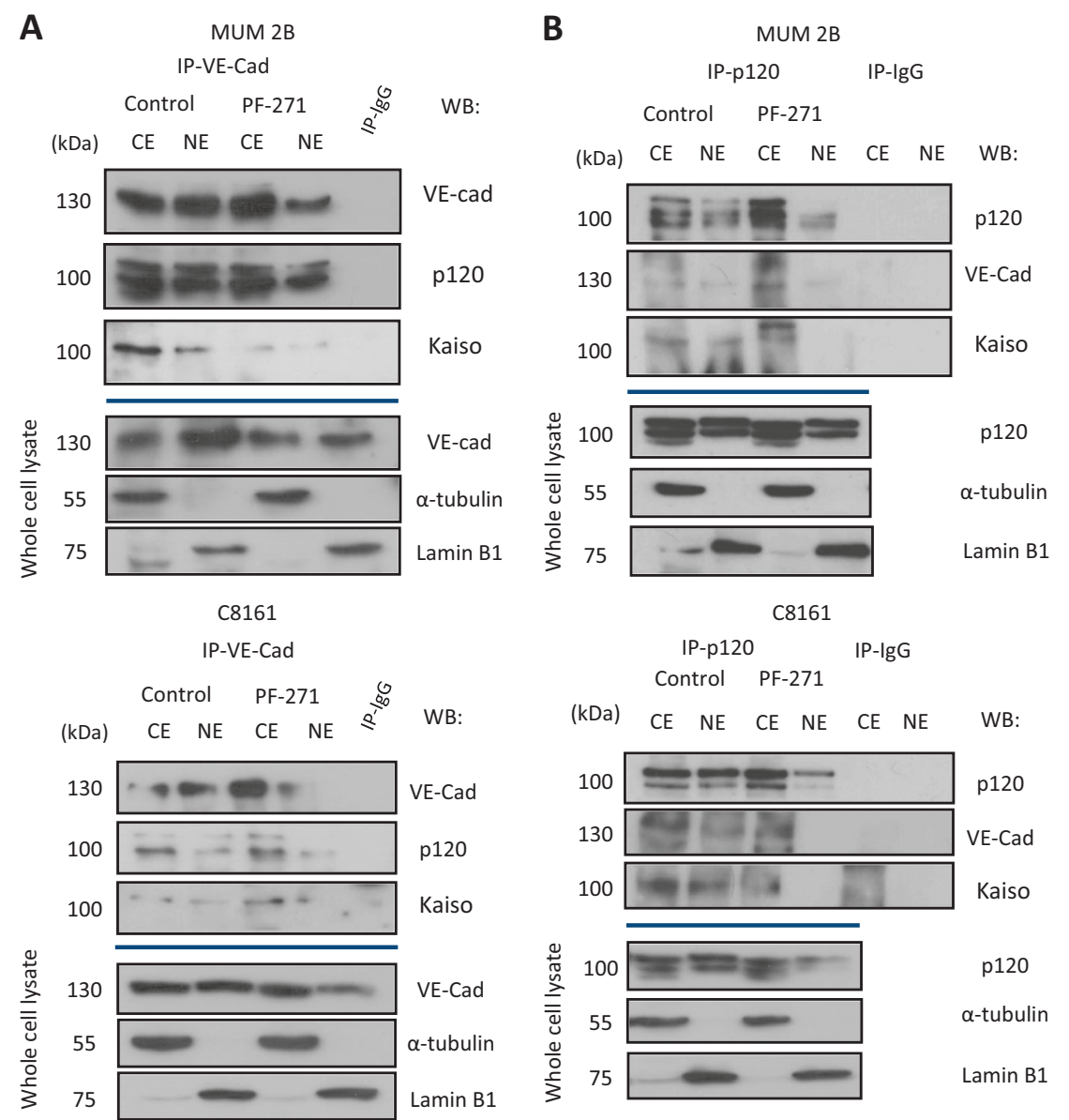

VEC is essential in the ability to form VM in aggressive melanoma cells.

\section{Discussion}

VM allows tumor perfusion owing to the specific capacity of malignant cells to form vessel-like networks which provide sufficient blood supply for tumor growth. An increasing number of studies have indicated that tumor angiogenesis not only includes endothelium-dependent vessels, but is also dependent on VM formed by cancer cells extracellular matrix. In fact, VM channels, mosaic blood vessels, and endothelial vessels coexist in malignant tumors and dynamically can exchange the status of each other according to fluctuations in the tumor microenvironment [6]. The mechanism allowing tumor cells the acquisition of this phenotype still remains to be fully elucidated. In ECs, VEC undergoes constitutive internalization driven by a unique endocytic motif that also serves as a p120catenin (p120) binding site. pVEC (Y658) serves as a signal to initiate the dissociation of p120-catenin from VEC allowing its internalization. Post-translational VEC modifications trigger junctional changes and VEC internalization. In particular, pVEC (Y658) is located within the p120-catenin binding region and has been shown to be the p120-catenin binding site responsible for VEC internalization [17]; inhibition of FAK activity prevents tumor metastasis by enhancing barrier function [17]. Furthermore, phosphorylation of Y658 has been reported to reduce p120 catenin binding, an important stabilizer of cadherins at the cell membrane [31]. FAK itself is phosphorylated at positions Y397 and Y576 in highly aggressive melanoma, but not in poorly aggressive melanoma cells; this posttranslational changes are indicative of a fully active FAK [16]. This autophosphorylated Y397 of FAK in highly aggressive melanoma cells could explain their high constitutively elevated pVECY658 levels. As shown in Fig. 4, alterations in both gene dosage and mRNA expression of FAK (PTK2) are frequently found in uveal melanoma patients $(57 \%$ of Uveal Melanoma display genetic alterations or upregulation of PTK2 mRNA) and, more interestingly, these variations are directly related with overall survival, highlighting the importance of FAK expression in human Uveal Melanoma progression.

Unexpectedly our results demonstrate that malignant melanoma cells that display VM capability constitutively do express high levels of pVEC (Y658). pVEC (Y658) and 

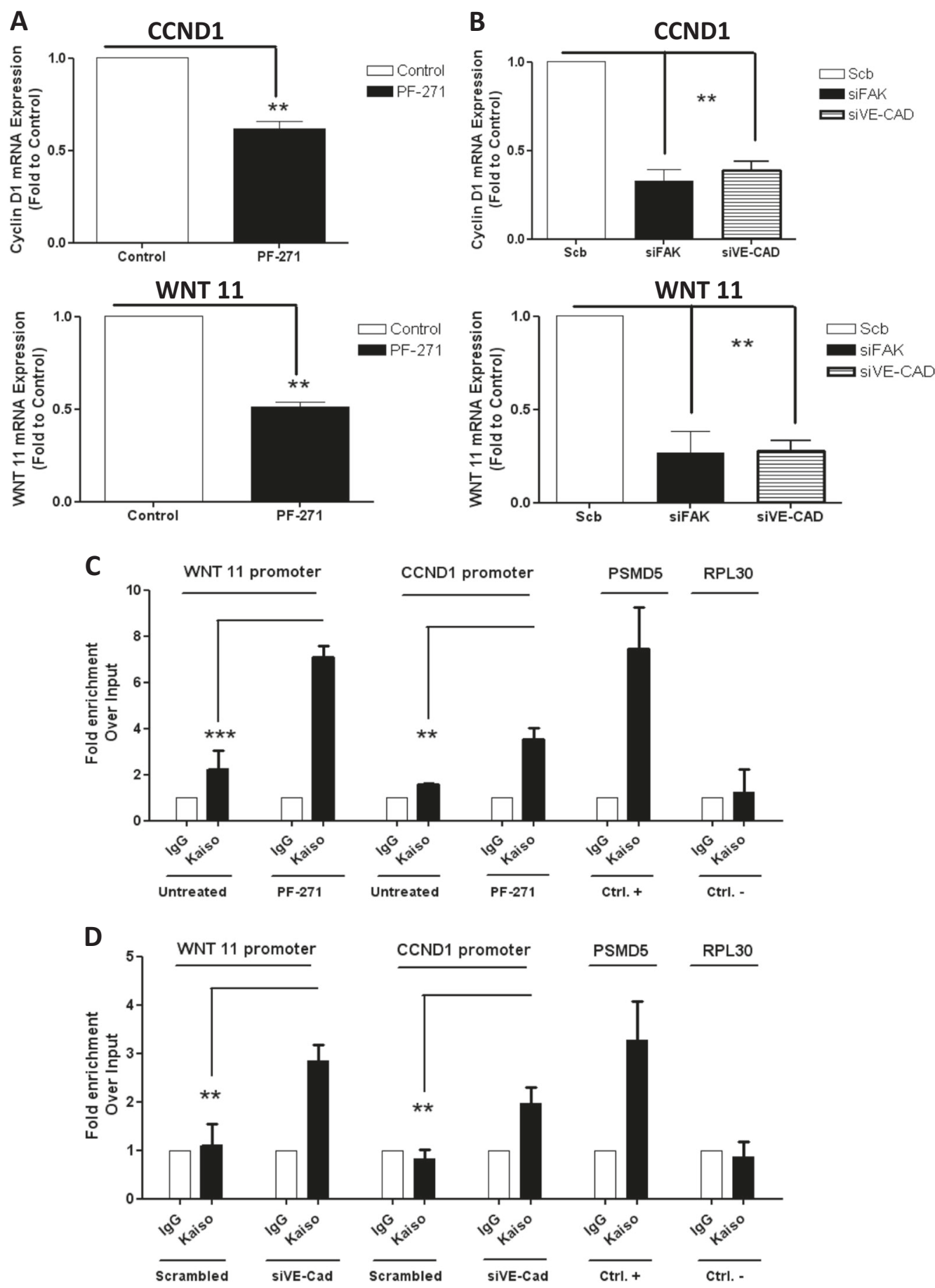

Fig. 7 pY658VE-cadherin represses the binding of kaiso to their target genes CCND1 and Wnt 11: a-b qPCR experiments with FAK-inhibitors or silencing FAK, VE-cadherin showed strong downregulation of kaiso-dependent genes (CCND1 and WNT 11). Statistical analyses were conducted using Graph Pad Prism software. Statistical significance was calculated using a Student's $t$ test (unpaired, two-tailed) with measurements from at least three independent trials. qPCR experiments with FAK-inhibitors or silencing FAK, VE-cadherin showed strong downregulation of kaisodependent genes (CCND1 and WNT 11). Statistical analyses were conducted using Graph Pad Prism software. Statistical significance was calculated using a Student's $t$ test (unpaired, two-tailed) with measurements from at least three independent experiments. c ChIP assay in MUM 2B cells at the Wnt11 promoter and CCND1 promoter with and without PF271 treatment. d Same assay with and without VE-CAD silencing. PSMD5 promoter is used as a positive control and RPL30 as irrelevant sequence for kaiso binding (negative control). Results are represented as fold enrichment over input. Asterisks denote significance in an unpaired $t$ test $\left({ }^{*} p<0.05,{ }^{* *} p<0.01,{ }^{* * *} p<0.001\right)$, and error bars denote SD 

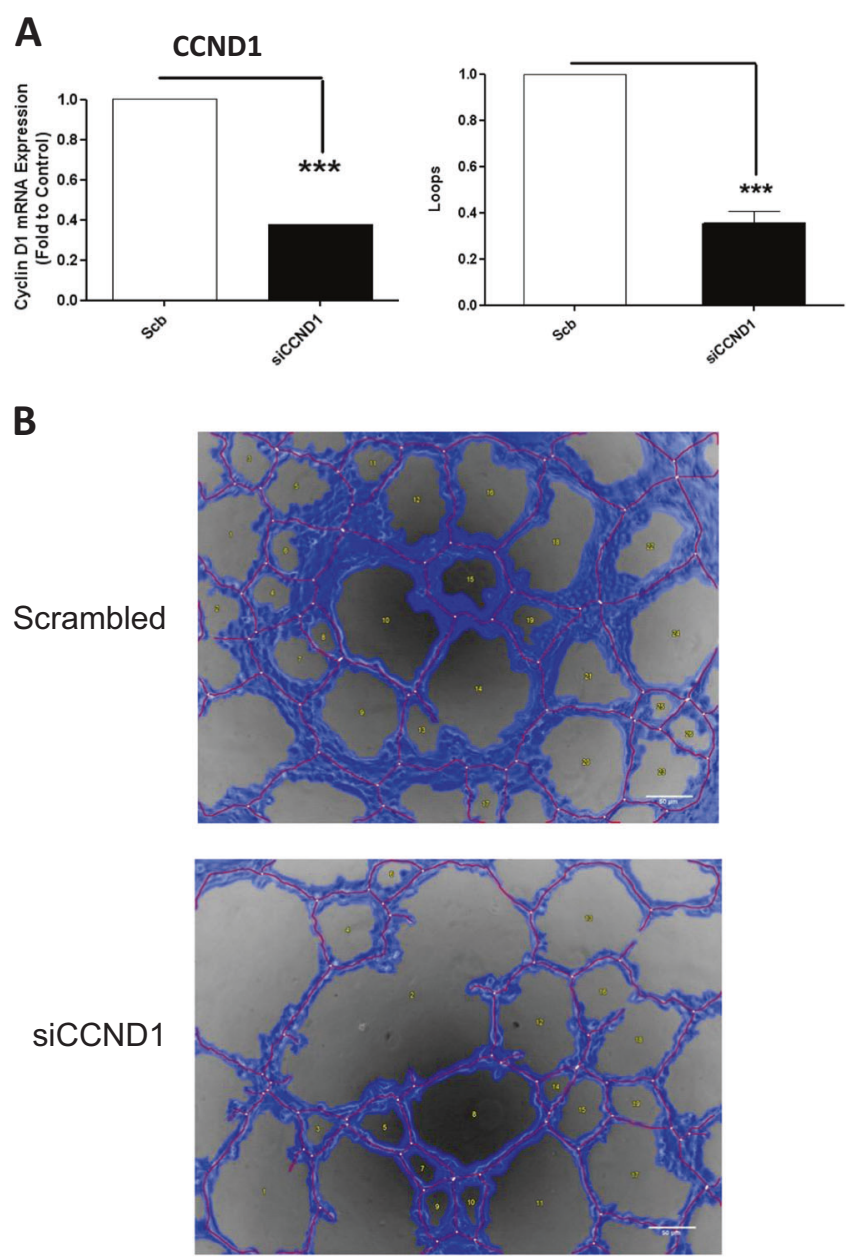

Fig. 8 pY658VE-cadherin represses the binding of kaiso to their target genes CCND1 and Wnt 11: c, d In vitro angiogenesis assay with Matrigel in MUM 2B showed the effect of siCCND1 or siWNT11, d images were acquired using an Olympus CKX41 microscope (bars 50

p120 remains in a complex, also present in the nucleus. The mechanistic explanation behind the persistence of this complex is still elusive and might be due to alterations in the p120 binding site to VEC or just as result of having a large population of pVEC (Y658) that stochiometrically saturates/disable p120 from detaching this complex. The mechanism by which pVEC go into the nucleus is not known. In these transformed cells a large population of pVEC is in permanent association with p120 and may use the same nuclear import mechanism as p120. Indeed, nuclear VEC is found in different tumor tissue samples (one example is represented in Fig. S4B, Human Protein Atlas, https://www.proteinatlas.org/ but the same subcellular localization was found in patients $2252,3535,1910$ (breast cancer), 1765, 3185 (lung cancer), 1787, 1158, 2142 (stomach cancer), 2931, 3041, and 2117 (colorectal cancer) and noteworthy, a similar cadherin, E-cadherin, implicated in intercellular adhesion between epithelial cells, has been also
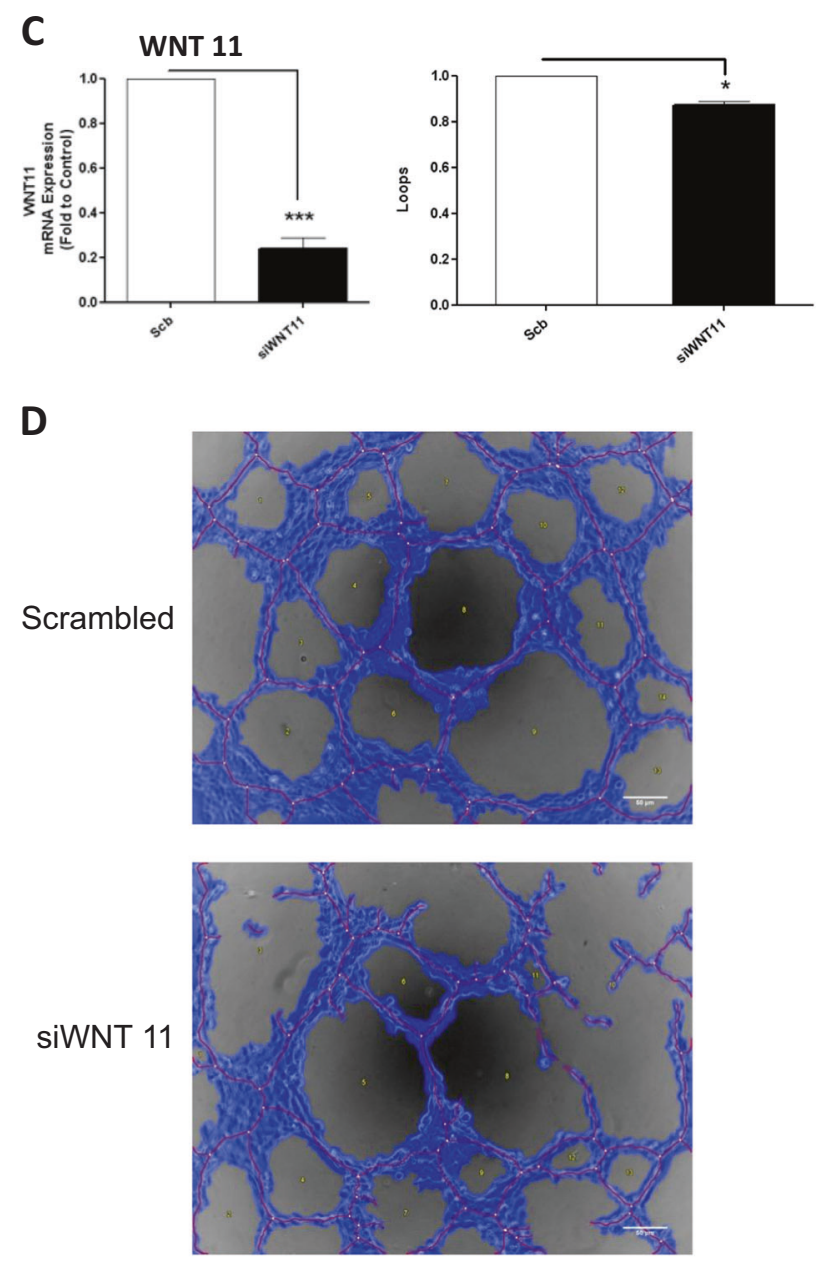

$\mu \mathrm{m})$ and the formation of tube-like structures was then quantified by Wimasis program. Each treatment was performed in triplicate, and the experiment was independently repeated at least three times. a, b siRNA of CCND1 and WNT 11 was confirmed by qPCR

found in the nucleus, and this subcellular location was directly associated with tumor grade and stage [32].

Because p120 binding to classical cadherins, both in the endothelium and in other tissues, is an important regulator of adherent junction dynamics, and responsible for the changing conditions in tumor microenvironment through modification of vessel leakiness, understanding other processes that may interfere with p120/VEC complex turnover remains an important area for research. There is significant disagreement over which sites may be phosphorylated under different conditions and whether p120 binding is disrupted [22, 31]. Moreover, the molecular insights of VEC dynamics in tumor cells have not been elucidated yet. In the current report we focus on the kinase FAK which has been reported to be critical for VEC internalization by targeting Y658 [17, 33]. Our results show that pVEC (Y658) phosphorylation is needed (but not sufficient) for malignant melanoma cells to undergo endothelial behavior. 
A

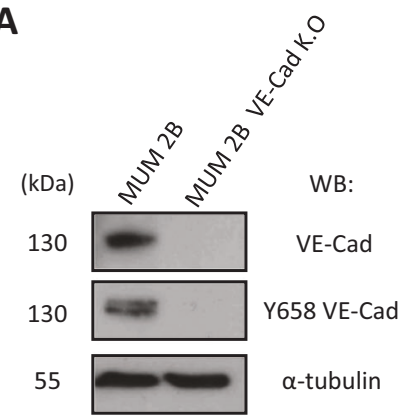

B

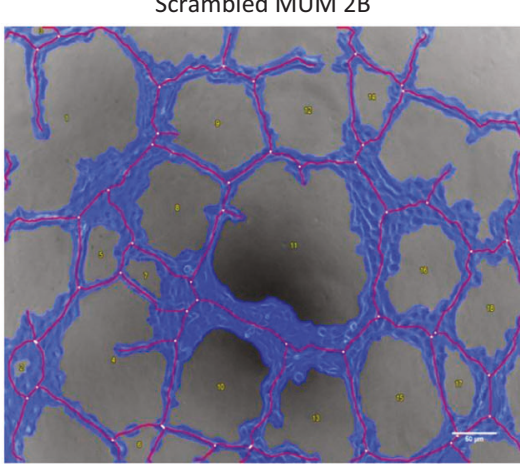

MUM 2B VE-Cad K.O + WT VE-Cad

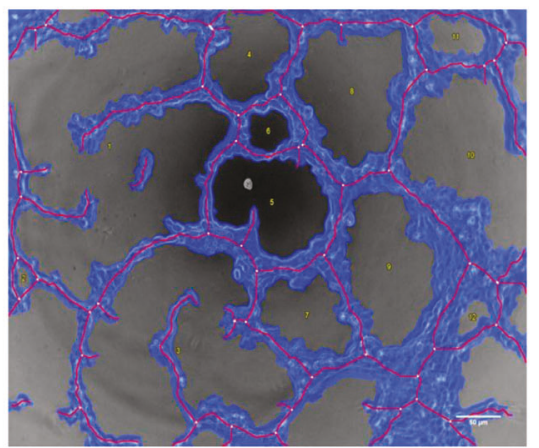

siVE-Cad MUM 2B

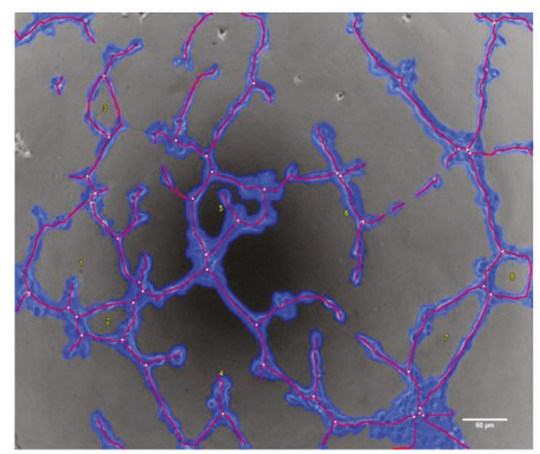

MUM 2B VE-Cad K.O + Y658F VE-Cad

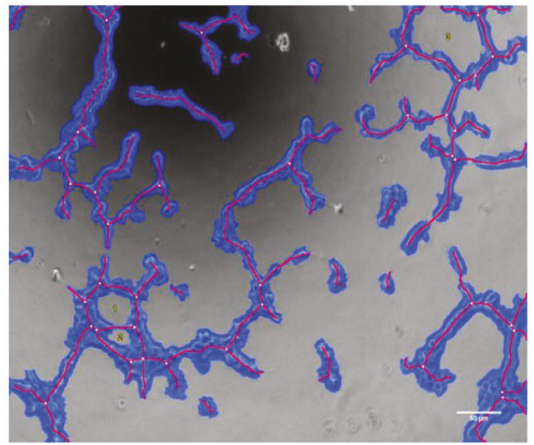

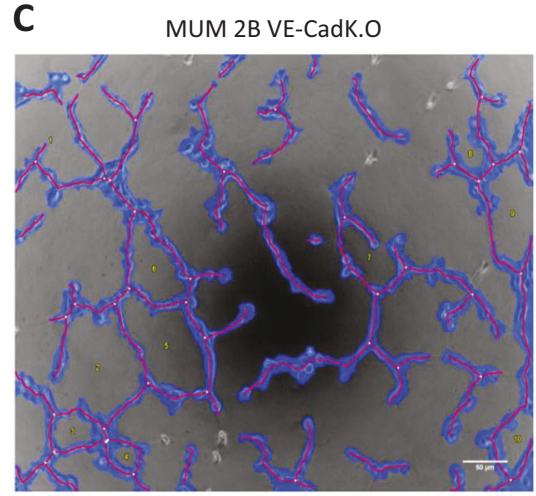

D

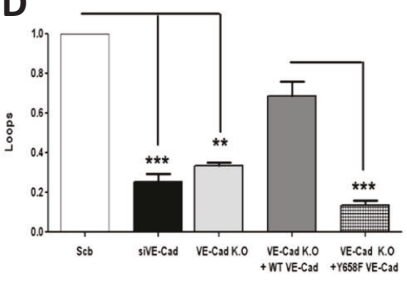

Fig. 9 VE-cadherin/Y658 phosphorylation is essential in the ability to form VM: a VE-cadherin and Y658 VE-cadherin expression in MUM 2B and MUM 2B VE-cadherin K.O, b, c In vitro angiogenesis assay with Matrigel in Scb, siVE-Cad, VE-Cad K.O, VE-Cad K.O+WT VECad construct, and VE-Cad K.O+Y658F VE-Cad construct in MUM 2B showed that VE-cadherin is essential for the formation of VM,

Intracellular trafficking of pVEC (Y658) is clearly altered as consequence of the persistence of the p120/pVEC (Y658) complex. In the nuclear compartment the protein complex persists by virtue of the phosphorylation of Y658: inhibition of the upstream kinase FAK or siFAK completely prevented complex formation and nuclear localization of VEC also diminished nuclear p120. P120 catenin plays a complex role in the nucleus and in the regulation of transcription factors. Similar to $\beta$-catenin, p120 can also localize to the nucleus and perform changes in nuclear signaling (for review see [34]). Daniel and Reynolds originally reported the association of p120 with the transcription factor kaiso [35]. Then we wondered for the consequences of having pVEC (Y658) as nuclear partner of p120 in kaiso-dependent gene expression. It is generally thought that kaiso binds promoter images were acquired using an Olympus CKX41 microscope (bars 50 $\mu \mathrm{m})$ and the formation of tube-like structures was then quantified by Wimasis program. Each treatment was performed in triplicate, and the experiment was independently repeated at least three times, d) VM formation as measured by the capacity to form loops quantified by the Wimasis software. ${ }^{* *} p<0.01, * * * p<0.001$

regions via two different mechanisms: a specific DNA consensus sequence [36], or through binding to methylated $\mathrm{CpG}$ dinucleotides [37]. While massively understudied compared to nuclear $\beta$-catenin signaling, available data suggest that kaiso acts primarily as a transcriptional repressor and that p120 binding activates transcription of kaiso targets via de-repression. In agreement, p120 overexpression reversed kaiso-mediated reduction in Siamois reporter activity, while p120 depletion had the opposite effect [38]. Our results place pVEC (Y658) as an essential player in the regulation of kaiso-dependent gene repression and connect the elevated amounts of pVEC (Y658) with malignancy through its ability to potentiate p120/kaiso complex formation, preventing kaiso-recruitment to specific KBS (kaiso binding sites). As result, elevated expression of 


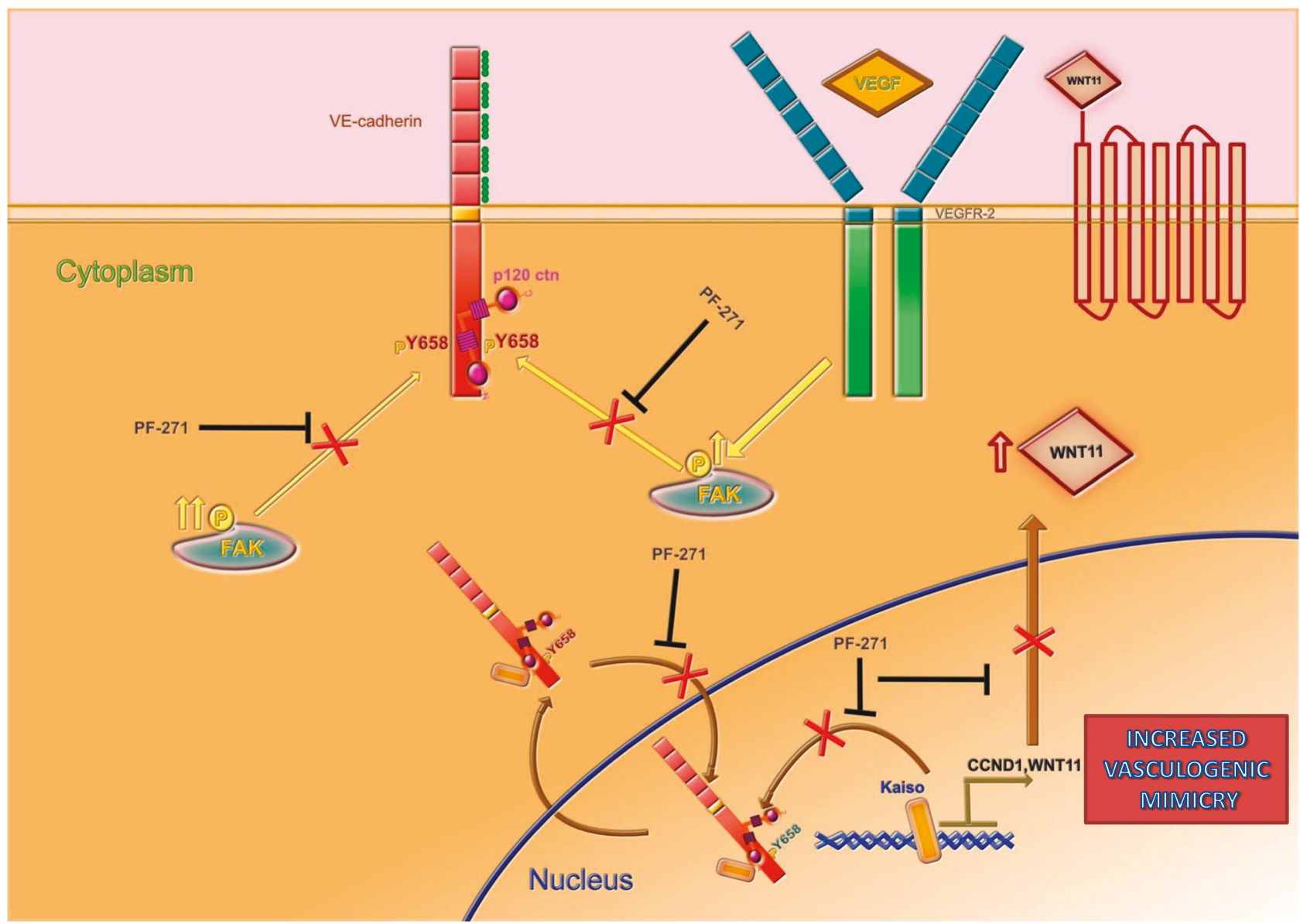

Fig. 10 Proposed model for the mechanism by which elevated pVEC expression leads to increased VM in malignant melanoma cells. Further details are explained under the discussion section

kaiso-repressed genes, such as wnt11 and CCDN1 poised the cell to more efficiently undergo VM. Wnts are morphogens with well-recognized functions during embryogenesis, and aberrant Wnt signaling has been demonstrated to be important in different settings carcinogenesis [39, 40]. WNT11 stimulates proliferation, migration, and invasion of cancer-derived cells, and CCDN1 (Cyclin D1) protein is highly expressed in patients with adenomatous polyps, primary colorectal adenocarcinoma, and familial adenomatous polyposis [41], and in mice bearing intestinal adenomas from multiple intestinal neoplasia [41]. Here we document a direct connection between post-translational modification of VEC and increased migration caused by Wnt 11 and CCDN1. In Fig. 10, we show a graphic abstract that summarized our current model: malignant melanoma cells, either cutaneous or uveal, express large amounts of FAK leading to constitutive pVEC (Y658). Subcellular localization for phosphorylated VEC is both the cytosol and the nucleus. Even in the absence of an external stimulus (as VEGFA treatment) pVEC is expressed and, contrary to what has been shown for ECs, p120-catenin remains in a complex with pVEC (Y658). More strikingly, to allow p120 nuclear localization, VEC must be phosphorylated by FAK whose inhibition promotes the accumulation of p120 in the cytosol. P120-catenin nuclear action on the transcription factor repressor kaiso requires the loading of pVEC (Y658) in the complex and prevention of VEC phosphorylation increases kaiso recruitment (then gene expression repression) of kaiso-dependent genes such as CCDN1 and WNT11. The presence of elevated pVEC (Y658) allows for upregulation of kaiso-dependent gene which in turn accelerate VM ability in these cells.

Together these findings define a mechanistic scenario to explain how VEC expression and more precisely pVEC (Y658) impact on cell's capability to undergo VM. It is interesting to note that non-phosphorylated VEC is not per se the trigger of VM: while the stability of VEC/p120catenin is essential to strength ECs junctions, the presence of elevated pVEC confers an additional plasticity to cancer cells that keeps them with intermediate traits between tumor and ECs, increasing the microenvironment complexity and allowing the presence of inflammation, hypoxia, and 
shortage of nutrients as a result of a deficient blood perfusion. These findings also shed light to new therapeutic opportunities targeting FAK to downregulate pVEC (Y658) levels and leading to abrogation of gene expression that amplifies VM formation by cancer cells.

Acknowledgements This work was supported by Junta de Andalucía, project of Excellence from Junta de Andalucía P10-CTS-0662, P12CTS-383, Spanish Ministry of Economy and Competitiveness SAF2012-40011-C02-01, SAF2015-70520-R, RTICC RD12/0036/ 0026, and CIBERONC ISCIII CB16/12/00421.

Author contributions DD-B performed the experiments, analyzed, interpreted the data, and designed the research; MF-C performed the experiments and analyzed the data; MI-R performed the experiments and interpreted the data; AG-D designed the research, interpreted and analyzed the data; AK interpreted and analyzed the data; FJO designed the research, interpreted and analyzed the data; DD-B, AG-D, and FJO wrote the manuscript.

\section{Compliance with ethical standards}

Conflict of interest The authors declare that they have no conflict of interest.

\section{References}

1. Ellis LM, Fidler IJ. Finding the tumor copycat. Therapy fails, patients don't. Nat Med. 2010;16:974-5.

2. Maniotis AJ, Folberg R, Hess A, Seftor EA, Gardner LM, Pe'er J, et al. Vascular channel formation by human melanoma cells in vivo and in vitro: vasculogenic mimicry. Am J Pathol. 1999;155:739-52.

3. Folberg R, Hendrix MJ, Maniotis AJ. Vasculogenic mimicry and tumor angiogenesis. Am J Pathol. 2000;156:361-81.

4. Hess AR, Seftor EA, Seftor RE, Hendrix MJ. Phosphoinositide 3kinase regulates membrane Type 1-matrix metalloproteinase (MMP) and MMP-2 activity during melanoma cell vasculogenic mimicry. Cancer Res. 2003;63:4757-62.

5. Bittner M, Meltzer P, Chen Y, Jiang Y, Seftor E, Hendrix M, et al. Molecular classification of cutaneous malignant melanoma by gene expression profiling. Nature. 2000;406:536-40.

6. Delgado-Bellido D, Serrano-Saenz S, Fernandez-Cortes M, Oliver FJ. Vasculogenic mimicry signaling revisited: focus on nonvascular VE-cadherin. Mol Cancer. 2017;16:65.

7. Dejana E, Tournier-Lasserve E, Weinstein BM. The control of vascular integrity by endothelial cell junctions: molecular basis and pathological implications. Dev Cell. 2009;16:209-21.

8. Hendrix MJ, Seftor EA, Meltzer PS, Gardner LM, Hess AR, Kirschmann DA, et al. Expression and functional significance of VE-cadherin in aggressive human melanoma cells: role in vasculogenic mimicry. Proc Natl Acad Sci USA. 2001;98: 8018-23.

9. Dejana E, Orsenigo F, Lampugnani MG. The role of adherens junctions and VE-cadherin in the control of vascular permeability. J Cell Sci. 2008;121(Pt 13):2115-22.

10. Vockel M, Vestweber D. How T cells trigger the dissociation of the endothelial receptor phosphatase VE-PTP from VE-cadherin. Blood. 2013;122:2512-22.

11. Chen XL, Nam JO, Jean C, Lawson C, Walsh CT, Goka E, et al. VEGF-induced vascular permeability is mediated by FAK. Dev Cell. 2012;22:146-57.
12. Schwock J, Dhani N, Hedley DW. Targeting focal adhesion kinase signaling in tumor growth and metastasis. Expert Opin Ther Targets. 2010;14:77-94.

13. Infante JR, Camidge DR, Mileshkin LR, Chen EX, Hicks RJ, Rischin D, et al. Safety, pharmacokinetic, and pharmacodynamic phase I dose-escalation trial of PF-00562271, an inhibitor of focal adhesion kinase, in advanced solid tumors. J Clin Oncol. 2012;30:1527-33

14. Haskell H, Natarajan M, Hecker TP, Ding Q, Stewart J Jr., Grammer JR, et al. Focal adhesion kinase is expressed in the angiogenic blood vessels of malignant astrocytic tumors in vivo and promotes capillary tube formation of brain microvascular endothelial cells. Clin Cancer Res. 2003;9:2157-65.

15. Lu C, Bonome T, Li Y, Kamat AA, Han LY, Schmandt R, et al. Gene alterations identified by expression profiling in tumorassociated endothelial cells from invasive ovarian carcinoma. Cancer Res. 2007;67:1757-68.

16. Hess AR, Postovit L-M, Margaryan NV, Seftor EA, Schneider GB, Seftor RE, et al. Focal adhesion kinase promotes the aggressive melanoma phenotype. Cancer Res. 2005;65:9851-60.

17. Jean C, Chen XL, Nam JO, Tancioni I, Uryu S, Lawson C, et al. Inhibition of endothelial FAK activity prevents tumor metastasis by enhancing barrier function. J Cell Biol. 2014;204:247-63.

18. Hsu PD, Scott DA, Weinstein JA, Ran FA, Konermann S, Agarwala V, et al. DNA targeting specificity of RNA-guided Cas9 nucleases. Nat Biotechnol. 2013;31:827.

19. Salmon P, Trono D. Production and titration of lentiviral vectors. Curr Protoc Neurosci. 2006; Chapter 4:Unit 4.21.

20. Rodriguez MI, Peralta-Leal A, O'Valle F, Rodriguez-Vargas JM, Gonzalez-Flores A, Majuelos-Melguizo J, et al. PARP-1 regulates metastatic melanoma through modulation of vimentin-induced malignant transformation. PLoS Genet. 2013;9:e1003531.

21. Hatanaka K, Simons M, Murakami M. Phosphorylation of VEcadherin controls endothelial phenotypes via p120-catenin coupling and Rac1 activation. Am J Physiol Heart Circ Physiol. 2011;300:H162-72.

22. Wessel F, Winderlich M, Holm M, Frye M, Rivera-Galdos R, Vockel M, et al. Leukocyte extravasation and vascular permeability are each controlled in vivo by different tyrosine residues of VE-cadherin. Nat Immunol. 2014;15:223-30.

23. Ward KK, Tancioni I, Lawson C, Miller NL, Jean C, Chen XL, et al. Inhibition of focal adhesion kinase (FAK) activity prevents anchorage-independent ovarian carcinoma cell growth and tumor progression. Clin Exp Metastasis. 2013;30:579-94.

24. Gavard J, Gutkind JS. VEGF controls endothelial-cell permeability by promoting the $\beta$-arrestin-dependent endocytosis of VEcadherin. Nat Cell Biol. 2006;8:1223-34.

25. Eliceiri BP, Paul R, Schwartzberg PL, Hood JD, Leng J, Cheresh DA. Selective requirement for Src kinases during VEGF-induced angiogenesis and vascular permeability. Mol Cell. 1999;4:915-24.

26. Xiao K, Garner J, Buckley KM, Vincent PA, Chiasson CM, Dejana E, et al. p120-Catenin regulates clathrin-dependent endocytosis of VE-cadherin. Mol Biol Cell. 2005;16:5141-51.

27. Daniel JM. Dancing in and out of the nucleus: p120(ctn) and the transcription factor Kaiso. Biochim Biophys Acta. 2007;1773: 59-68.

28. Pozner A, Terooatea TW, Buck-Koehntop BA. Cell-specific Kaiso (ZBTB33) regulation of cell cycle through cyclin D1 and cyclin E1. J Biol Chem. 2016;291:24538-50.

29. Kim SW, Park JI, Spring CM, Sater AK, Ji H, Otchere AA, et al. Non-canonical Wnt signals are modulated by the Kaiso transcriptional repressor and p120-catenin. Nat Cell Biol. 2004;6: 1212-20.

30. Spring CM, Kelly KF, O'Kelly I, Graham M, Crawford HC, Daniel JM. The catenin p120ctn inhibits Kaiso-mediated 
transcriptional repression of the beta-catenin/TCF target gene matrilysin. Exp Cell Res. 2005;305:253-65.

31. Nanes BA, Chiasson-MacKenzie C, Lowery AM, Ishiyama N, Faundez V, Ikura M, et al. p120-catenin binding masks an endocytic signal conserved in classical cadherins. J Cell Biol. 2012;199:365-80.

32. Su YJ, Chang YW, Lin WH, Liang CL, Lee JL. An aberrant nuclear localization of E-cadherin is a potent inhibitor of Wnt/ beta-catenin-elicited promotion of the cancer stem cell phenotype. Oncogenesis. 2015;4:e157.

33. Zhao X, Peng X, Sun S, Park AY, Guan JL. Role of kinaseindependent and -dependent functions of FAK in endothelial cell survival and barrier function during embryonic development. J Cell Biol. 2010;189:955-65.

34. McCrea PD, Maher MT, Gottardi CJ. Nuclear signaling from cadherin adhesion complexes. Curr Top Dev Biol. 2015;112:129-96.

35. Daniel JM, Reynolds AB. The cateninp120(ctn) interacts with Kaiso, a novel BTB/POZ domain zinc finger transcription factor. Mol Cell Biol. 1999;19:3614-23.

36. Daniel JM, Spring CM, Crawford HC, Reynolds AB, Baig A. Thep120(ctn)-binding partner Kaiso is a bi-modal DNA-binding protein that recognizes both a sequence-specific consensus and methylated $\mathrm{CpG}$ dinucleotides. Nucleic Acids Res. 2002;30:2911-9.

37. Prokhortchouk A, Hendrich B, Jorgensen H, Ruzov A, Wilm M, Georgiev G, et al. The p120 catenin partner Kaiso is a DNA methylation-dependent transcriptional repressor. Genes Dev. 2001;15:1613-8.

38. Park JI, Kim SW, Lyons JP, Ji H, Nguyen TT, Cho K, et al. Kaiso/ p120-catenin and TCF/beta-catenin complexes coordinately regulate canonical Wnt gene targets. Dev Cell. 2005;8:843-54.

39. Ouko L, Ziegler TR, Gu LH, Eisenberg LM, Yang VW. Wnt11 signaling promotes proliferation, transformation, and migration of IEC6 intestinal epithelial cells. J Biol Chem. 2004;279:26707-15.

40. Mori H, Yao Y, Learman BS, Kurozumi K, Ishida J, Ramakrishnan SK, et al. Induction of WNT11 by hypoxia and hypoxiainducible factor-1alpha regulates cell proliferation, migration and invasion. Sci Rep. 2016;6:21520.

41. Zhang T, Nanney LB, Luongo C, Lamps L, Heppner KJ, DuBois $\mathrm{RN}$, et al. Concurrent overexpression of cyclin D1 and cyclindependent kinase $4(\mathrm{Cdk} 4)$ in intestinal adenomas from multiple intestinal neoplasia (Min) mice and human familial adenomatous polyposis patients. Cancer Res. 1997;57:169-75. 